\title{
Terpene tail-to-head polycyclization mediated by small molecule catalysts: a weakly-coordinating anion approach
}

\author{
Jessica E. Burch, Alex L. Bagdasarian, Tanin Hooshmand, \& Hosea M. Nelson* \\ Department of Chemistry and Biochemistry, University of California, Los Angeles, Los \\ Angeles, CA 90095, USA. \\ *Correspondence to: hosea@chem.ucla.edu
}

\begin{abstract}
Biomimetic total synthesis has played a pivotal role in the development of synthetic organic chemistry. In particular, efforts aimed at mimicking the head-to-tail (HT) cation $-\pi$ cyclization cascades invoked in terpene biosynthesis, such as those catalyzed by type-II cyclases, have led to a multitude of new synthetic methods, chemical concepts, and total syntheses over the past century. Conversely, synthetic methodology that mimics tail-to-head $(\mathrm{TH})$ cation $-\pi$ cyclization cascades, mediated by $\mathrm{Mg}^{2+}$ type-I terpene cyclases, remains elusive in organic synthesis, despite key roles in the biosynthesis of privileged therapeutic molecules such as taxol and artemesinin. Here we report that $\mathrm{Li}^{+}$/weakly-coordinating anion (WCA) salts catalyze the $\mathrm{TH}$ polycyclization of linaloyl fluoride, leading to high-yielding mixtures of polycyclic terpene natural products including cedrenes, cadinadiene, epizonarene, and $\delta$-selinene. The examples reported herein represent early examples of small molecule-catalyzed TH polycyclization reactions enabling the shortest (formal) total synthesis of $( \pm$-artemisinin. Moreover we apply this strategy to the diterpene geranyllinaloyl fluoride, resulting in a two-step total synthesis of the tricyclic core of the gersemiols (named here as $\alpha$-gersemiene), a recently discovered class of marine diterpenoid natural products.
\end{abstract}

\section{Introduction}

For nearly 70 years, the study of terpene biosynthesis has inspired scientists across a wide array of disciplines. ${ }^{1-6}$ From enzymology and biophysics to computational and synthetic chemistry, many of the fundamental principles driving modern chemical science are rooted in studies of these remarkable enzymatic processes. ${ }^{7}$ Early investigation of triterpene stereochemistry by Stork and Eschenmoser led to the initially controversial hypothesis that cyclase enzymes generate reactive carbocations to yield steroid cores through cation $-\pi$ cyclization cascades. $^{3}$ This groundbreaking hypothesis ultimately inspired the development of small-molecule catalysts capable of producing polydecalin and steroid-like compounds with high levels of stereocontrol. Indeed, since the 1960s, Johnson, Corey, Yamamoto, Overman, and others have demonstrated that employment of a biomimetic head-to-tail (HT) cation- $\pi$ cyclization strategy in total synthesis provides a powerful platform to access polydecalin natural products. ${ }^{8-12}$

These classic studies highlight the value of using terpene biosynthesis as a sharpening stone for chemical synthesis and enzymology. However this symbiotic relationship continues to be confined to HT processes that mimic type-II terpene cyclases that produce polydecalin frameworks (e.g. 1, Figure 1a) from linear isoprenoids (2). 
Conversely, polycyclization reactions that mimic $\mathrm{Mg}^{2+}$-dependent type-I terpene cyclases remain understudied in synthetic organic chemistry; this is despite forging a larger and more structurally-diverse subset of polycyclic terpenes, including medicinally privileged natural products such as artemisinin and taxol. ${ }^{7}$ These processes, originally coined tailto-head (TH) cyclization by Shenvi and Pronin, proceed enzymatically via $\mathrm{Mg}^{2+}$ mediated ionization of a phosphate head group (e.g., 3, Figure 1b), followed by attack of an isoprenyl tail (e.g., 3-6) to ultimately form macrocyclic, medium, or small rings (5). ${ }^{13-}$ ${ }^{15}$ The savagely acidic and electrophilic carbocation intermediates in these processes (e.g. 6) are prone to rapid $\mathrm{E} 1$ elimination or $\mathrm{S}_{\mathrm{N}} 1$ reactions in bulk solvent, often precluding productive polycyclization in a synthetic setting. This stands in stark contrast to the wellstudied HT processes where the bond-forming events occur through low-energy

a) Head-to-tail (HT) cationic cyclizations

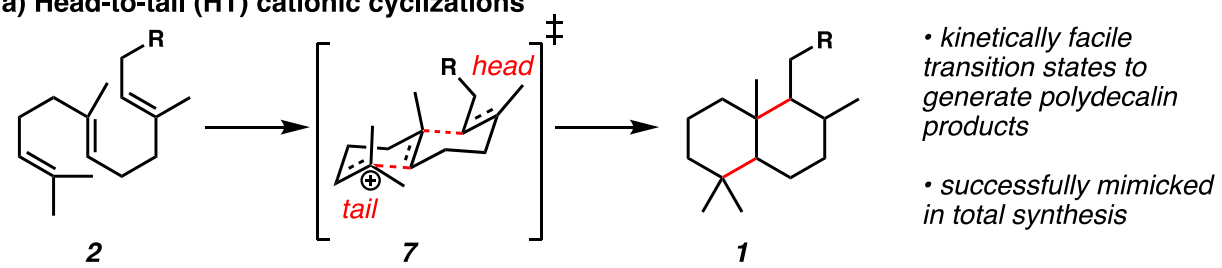

b) Tail-to-head (TH) cationic cyclizations

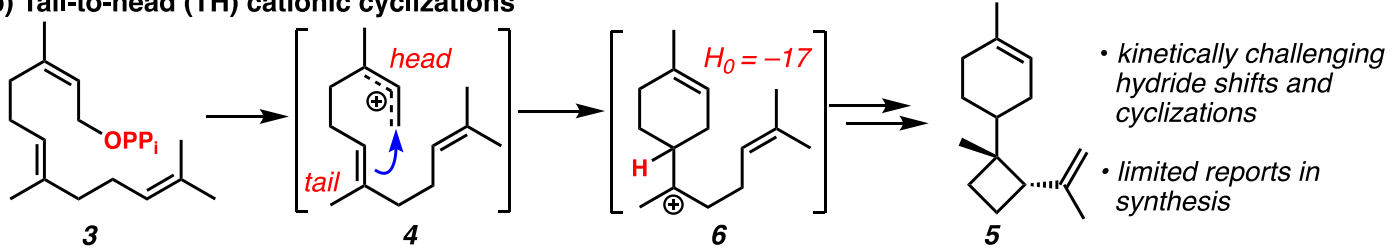

c) Stoichiometric sequestered anion approach (2012)

d) Supramolecular encapsulation approach (2018)

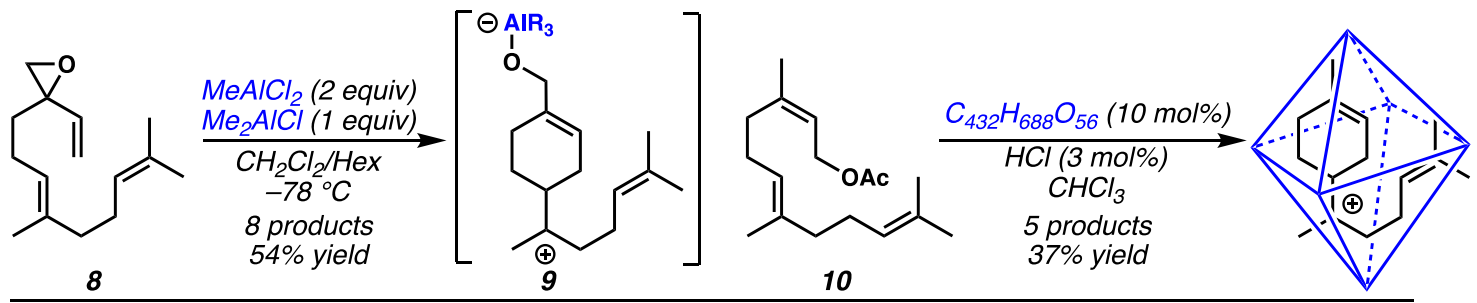

This work:

e) TH cationic cyclization using lithium-WCA salt catalysis

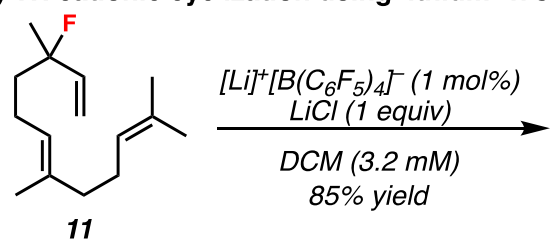

f) Extension towards diterpene cyclizations

17

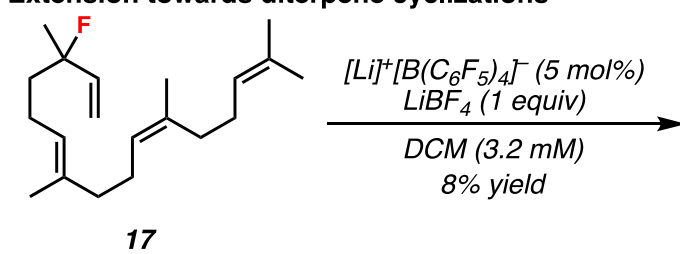<smiles>C=C(C)[C@H]1CC[C@H](C)[C@H]2CCC(C)=C[C@H]12</smiles>

12<smiles>CC1=CC[C@@]23CCC(C)[C@H](CC2)C(C)(C)[C@H]13</smiles>

$13 / 14$<smiles>CC1=CC2=C(C(C)[CH+]C(C)C3=CC4=C(C)CCCC4(C)CC3)CC[C@@H](C)[C@H]2CC1</smiles>

15
16<smiles>CC1=C2CC[C@H]3C(C)=C3CC[C@]3(C)CCC=C(C1)[C@@]23C</smiles>

18, $\alpha$-gersemiene

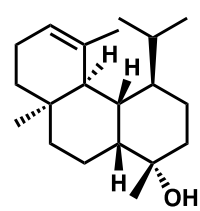

29, gersemiol C

Figure 1. Previous and current examples of cationic terpene cyclizations. 
polydecalin transition states (e.g. 7, Figure 1a), allowing for rapid polycyclization that often outcompetes deleterious $\mathrm{E} 1$ or $\mathrm{S}_{\mathrm{N}} 1$ pathways.

Many early efforts to synthesize sesquiterpenes through biomimetic $\mathrm{TH}$ polycyclization have been reported, often resulting in low-yielding complex mixtures largely comprised of monocyclic bisabolenes. ${ }^{16-19}$ Reports from Shenvi and coworkers demonstrated that treatment of an unnaturally-occurring farnesene oxide species (8, Figure 1c) with stoichiometric Lewis acid yields a synthetically relevant mixture of biand tricyclic sesquiterpenoids through a putative zwitterionic intermediate (9). ${ }^{13}$ Recently, the first example of non-enzymatic, catalytic TH cyclization was reported by Tiefenbacher and coworkers, who employed a supramolecular cluster to engage farnesyl acetate (10, Figure 1d) affording an array of polycyclic sesquiterpene products. The product selectivity observed in this seminal example of catalytic $\mathrm{TH}$ cyclization, in particular the formation of $\delta$-selinene (16), was attributed to encapsulation by the supramolecular assembly. ${ }^{14,15}$ Here we diverge in approach from these seminal reports and describe the utilization of weakly-coordinating anion (WCA) catalysis to achieve the biomimetic conversion of sesquiterpene fluoride $\mathbf{1 1}$ (Figure 1e) to high yielding mixtures of polycyclic sesquiterpene natural products (12-16). Notably, cadinadiene (12), the trans-decalin variant of amorphadiene and a reported biosynthetic precursor of artemisinin, is formed in this reaction, constituting a 7-step formal total synthesis of the bioactive natural product. ${ }^{20}$ Moreover, we report proof-of-principle that this strategy is amenable to diterpene total synthesis, as we demonstrate that geranyllinaloyl fluoride (17, Figure 1f) can be converted directly to the tricyclic diterpene, $\alpha$-gersemiene (18) using this simple catalytic system.

\section{Results and Discussion}

At the outset of our efforts, we hypothesized that the use of WCAs would allow for the generation of persistent carbocations with sufficient lifetimes to partake in polycyclization events before fast, counteranion-mediated-E1 elimination or trapping by solvent. ${ }^{21-23}$ Previous reports from our lab have demonstrated the competency of $\mathrm{R}_{3} \mathrm{Si}^{+} /$WCA-derived catalysts in generating long-lived carbocationic species that engage in intermolecular $\mathrm{C}-\mathrm{H}$ insertion reactions. ${ }^{24}$ Hence, we hypothesized that allylic fluoride 11, (Figure 1e) could be readily ionized under analogous $\mathrm{R}_{3} \mathrm{Si}^{+} / \mathrm{WCA}$ conditions to generate an allylic carbocation (4, Figure 1 b) poised to undergo rapid 1,6-cyclization to generate the bisabolyl cation (6) ${ }^{25,26}$ Despite having a Hammett acidity of $c a . \leq-17$, we posited that this carbocation would have sufficient lifetime, when paired with a WCA, to undergo subsequent polycyclization without the formation of bisabolenes through E1 elimination. ${ }^{27}$

To validate our hypothesis, $(E)$-nerolidyl fluoride (11) was chosen as the model substrate (Figure 1e). Exposing fluoride 11 to triethylsilane and a catalytic amount of commercially available trityl tetrakis(pentafluorophenyl)borate, we observed lowyielding formation of $\alpha$-cedrene (13), epi- $\alpha$-cedrene (14), and cadinadiene (12) in addition to an intractable mixture of hydrocarbon products (Table 1, entry 1). Gratifyingly, these polycyclic sesquiterpenes arise from multiple hydride shifts and cyclization events subsequent to the formation of the bisabolyl cation, supporting our mechanistic hypothesis. ${ }^{25,26}$ Discouraged by the lack of selectivity and poor efficiency, 
we posited that the incompatibility of $\mathrm{R}_{3} \mathrm{Si}^{+}$/WCA catalysts with dichloromethane and olefinic substrates was responsible for the poor reaction outcome. ${ }^{28}$ These findings led us to explore the use of $\mathrm{Li}^{+} / \mathrm{WCA}$ catalysts with $(E)$-nerolidyl fluoride (11) to potentially attenuate any decomposition observed when utilizing $\mathrm{R}_{3} \mathrm{Si}^{+} /$WCA. Unfortunately, use of our reported $\mathrm{Li}^{+} / \mathrm{WCA}$ catalytic conditions, featuring the in situ generation of $[\mathrm{Li}]^{+}\left[\mathrm{B}\left(\mathrm{C}_{6} \mathrm{~F}_{5}\right)_{4}\right]^{-}$through combination of LiHMDS and $\left[\mathrm{Ph}_{3} \mathrm{C}\right]^{+}\left[\mathrm{B}\left(\mathrm{C}_{6} \mathrm{~F}_{5}\right)_{4}\right]^{-}$, resulted in

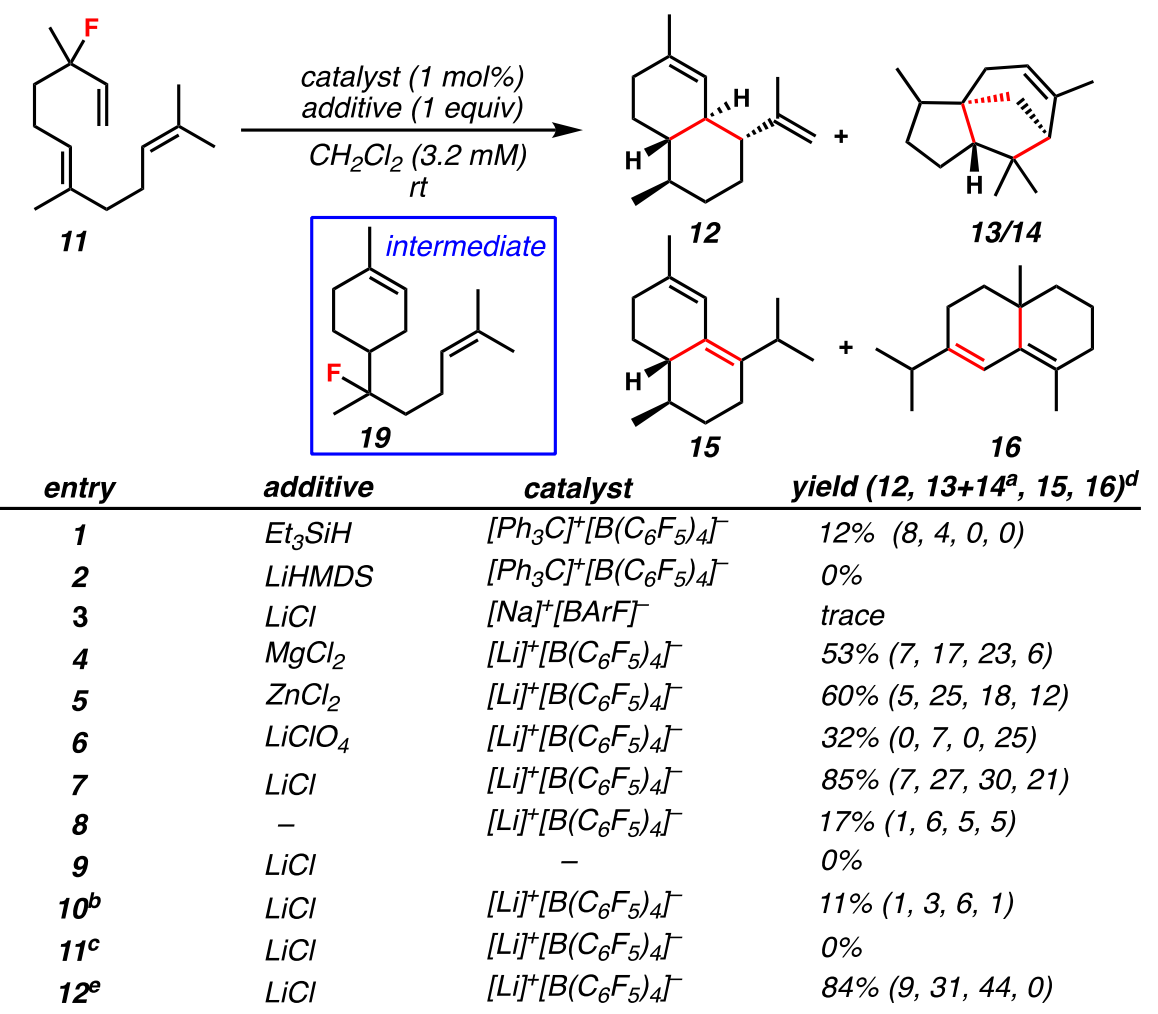

Table 1. Optimization table. ${ }^{\mathrm{a}}$ Two diastereomers estimated from crude ${ }^{1} \mathrm{H}$ NMR $(1: 1$ d.r. $) .{ }^{\mathrm{b}}$ Reaction performed at $-40{ }^{\circ} \mathrm{C} .{ }^{\mathrm{c}}$ Reaction performed at $40{ }^{\circ} \mathrm{C} .{ }^{\mathrm{d}}$ Yield calculated by NMR. ${ }^{\mathrm{e}}$ Utilizing bisabolyl fluoride 19 as starting material.

premature deprotonation of the intermediate carbocation, yielding a mixture of linear and monocyclic elimination products (entry 2) ${ }^{29}$ However, we were gratified to find that pairing mild inorganic bases with metal/WCA salts yielded polycyclic products, albeit in low yields (entries $3-5$ ). Utilization of stoichiometric $\mathrm{LiClO}_{4}$ was found to produce the cyclized terpene products in reduced yield, presumably due to promiscuous oxidative reactivity, as aromatic species such as cadalene were observed in the reaction mixture (entry 6, see Supporting Information). It was ultimately discovered that pre-formed $[\mathrm{Li}]^{+}\left[\mathrm{B}\left(\mathrm{C}_{6} \mathrm{~F}_{5}\right)_{4}\right]^{-}(1 \mathrm{~mol} \%)$ used in combination with stoichiometric $\mathrm{LiCl}$ (1 equivalent) provided a remarkable $85 \%$ combined yield of five known sesquiterpene natural products: cadinadiene (12), $\alpha$-cedrene (13), epi- $\alpha$-cedrene (14), epizonarene $(15)$, and $\delta$ selinene (16) (entry 7). ${ }^{30}$ Control reactions were performed without stoichiometric LiCl resulting in reduced yields (entry 8). Use of $\mathrm{LiCl}$ in the absence of catalyst resulted in no reaction (entry 9). Performing the reaction at reduced temperature failed to ionize the substrate, while elevated temperatures resulted in formation of bisabolenes (entries $10-$ 11). Interestingly, careful monitoring of the reaction by NMR and GC-FID revealed that bisabolyl fluoride (19) is an intermediate in this transformation. The origin of the fluorine 
atom in bisabolyl fluoride (19) is undetermined; however, reactive carbocations are known to undergo exchange reactions with typically inert molecules. ${ }^{31}$

The biosynthetic pathway for $\delta$-selinene (16) is proposed to proceed via an initial 1,10-cyclization of $(E, E)$-farnesyl pyrophosphate $(\mathbf{2 1}$, Figure $2 \mathrm{a})$ to generate germacrene A (22) which subsequently undergoes ring-closing to forge the selinene core. ${ }^{32}$ The direct generation of a 10 -membered carbocycle in our small molecule-catalyzed system seemed unlikely, given the potential for competitive formation of a 6-membered ring (i.e. 4, Figure 1 b vs. 23, Figure 2a). Interestingly, upon subjection of bisabolyl fluoride (19) to the optimized reaction conditions, we found high conversion (84\%) to cadinadiene (12), $\alpha$-cedrenes (13/14), and epizonarene (15), however no $\delta$-selinene (16) was detected (entry 12, Table 1). The lack of formation of $\delta$-selinene (16) from bisabolyl fluoride (19) supports the hypothesis that a biomimetic 1,10-cyclization could be occurring in our system. To probe this hypothesis, dihydronerolidyl fluoride (25, Figure 2 b), lacking the central olefin required to undergo a 1,6-cyclization, was subjected to the optimized reaction conditions. In the event, the 10 -membered carbocyclic product $\mathbf{2 6}$ was formed in $57 \%$ yield. Taken together, these experiments support biomimetic formation of $\delta$-selinene (16) through a 1,10-cyclization process without preorganization within an enzyme active site or supramolecular capsule. ${ }^{32}$ While enzyme-mediated medium size ring formation from linear precursors is commonly invoked in biosynthesis, analogous catalytic transformations remain rare in synthetic chemistry. ${ }^{33-35}$

a) 1,10-cyclization in biosynthesis of selinenes

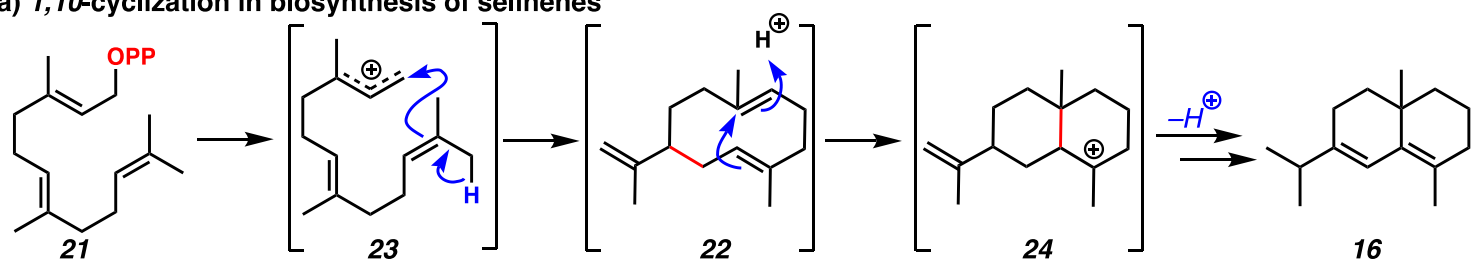

b) Dihydronerolidyl fluoride cyclization<smiles>C=CC(C)(F)CCCC(C)CCC=C(C)C</smiles>

c) Cyclization of geranyllinaloyl fluoride

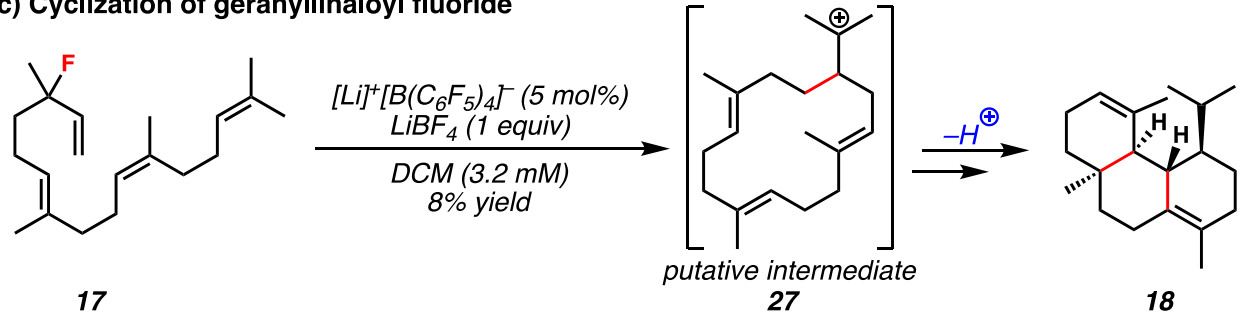

Figure 2. Evaluating cyclizations generating medium- and large-carbocyclic cationic intermediates.

Intrigued by this enzyme-free, medium-sized ring forming reaction, we wondered whether this catalytic strategy could be utilized in the biomimetic syntheses of more complex polycyclic diterpenes, which often proceed through medium- or macro-sized rings in nature. For example, taxadiene, the oxidative precursor of taxol, originates from 
a tail-to-head cyclization of geranylgeranyl pyrophosphate to forge the 14-membered cembrenyl carbocation (27). ${ }^{36}$ To the best of our knowledge, biomimetic approaches to taxadiene have not been reported, presumably due in part to the high entropic cost of forging such rings outside the confines of an enzyme. ${ }^{7}$ Interestingly, we found that treatment of geranyllinaloyl fluoride (17, Figure 2c) with stoichiometric $\mathrm{LiBF}_{4}$ and catalytic $\mathrm{Li}^{+} / \mathrm{WCA}$ catalyst led to formation of $\alpha$-gersemiene (18) in $8 \%$ yield. It is worthy to note that this putative natural product has also been observed in the acidpromoted cyclization of isocembrene, ${ }^{37}$ suggesting that the cembrenyl carbocation (27) is an intermediate in this process as well. $\alpha$-Gersemiene (18), is posited to be the biosynthetic precursor of the gersemiols, a class of recently isolated natural products from the soft coral species Gersemia fruiticosa $\left(\mathbf{2 8}-\mathbf{2 9}\right.$, Figure 1f). ${ }^{38}$

\section{Conclusion}

In summary, we report $\mathrm{Li}^{+} / \mathrm{WCA}$-catalyzed tail-to-head, biomimetic cation- $\pi$ cyclization reactions of sesquiterpenes and diterpenes. To the best of our knowledge, these are some of the first small molecule-catalyzed TH polycyclizations reported. This vertical advance in the field enables the 2-step syntheses of several sesquiterpene cores, including $\alpha$-cedrene (13), epi- $\alpha$-cedrene (14), cadinadiene (12), epizonarene (15), and $\delta$ selinene (16). Importantly, the synthesis of cadinadiene (12) represents the shortest (formal) total synthesis of artemesinin. ${ }^{20}$ We also report the first small moleculecatalyzed, biomimetic TH cyclization to forge a polycyclic diterpene, gersemiane A (18), albeit in modest yield. Additionally, we observe competitive formation of a 10membered terpene intermediate despite the availability of a more facile pathway leading to a 6-membered ring. Furthermore, given the competency of bisabolyl fluoride (19, Table 1) in the formation of several complex sesquiterpenes under our reaction conditions, our findings suggest that non-stop cyclization is not necessary for mimicking tail-to-head pathways in a synthetic setting. ${ }^{13}$ Taken together, these findings may offer a shift in the biomimetic paradigm that could ultimately unlock tail-to-head cyclization for use in practical organic synthesis. Akin to classic studies in steroid synthesis, we surmise that advancements in TH methodology will refine our understanding of the role of terpene cyclases in biosynthesis. ${ }^{39,40}$ Thus, developing TH cyclization methodology offers exciting possibilities in an underexplored area.

\section{Acknowledgements}

Financial support for this work was generously provided by the David and Lucile Packard Foundation (to H.M.N.), the Alfred P. Sloan Foundation (to H.M.N.), the Pew Charitable Trusts (to H.M.N), the NIH-NIGMS (R35 GM128936 to H.M.N.), and the National Science Foundation (DGE-1650604 to J.E.B.). A.L.B. thanks the Christopher S. Foote Fellowship for funding. The authors thank the UCLA Molecular Instrumentation Center for NMR and mass spectroscopy instrumentation. The authors would like to thank Prof. Dean Tantillo (University of California, Davis) and Dr. Johnny Gordon for useful discussions.

\section{Author Contributions}

H.M.N. conceived the idea and supervised the project. J.E.B. and A.L.B. performed the described experiments and analysed the data. T. H. assisted in sample purification. H.M.N., J.E.B., and A.L.B. co-wrote the manuscript.

The authors declare no competing financial interest. 


\section{References}

1. Ruzicka, L., Eschenmoser, A. \& Heusser, H. The isoprene rule and the biogenesis of terpenic compounds. Experientia 9, 357-367 (1953).

2. Stork, G. \& Burgstahler, A. W. The stereochemistry of polyene cyclization. J. Am. Chem. Soc. 77, 5068-5077 (1955).

3. Eschenmoser, A., Ruzicka, L., Jeger, O. \& Arigoni, D. A stereochemical interpretation of the biogenetic isoprene rule for the triterpenes. Helv. Chim. Acta 38, 1890-1904 (1955).

4. Maimone, T. J. \& Baran, P. S. Modern synthetic efforts toward biologically active terpenes. Nat. Chem. Biol. 3, 396-407 (2007).

5. Alleman, R. K. Chemical wizardry? The generation of diversity in terpenoids

biosynthesis. Pure Appl. Chem. 80, 1791-1798 (2008).

6. Tantillo, D. J. Biosynthesis via carbocations: theoretical studies on terpene

biosynthesis. Nat. Prod. Rep. 28, 1035-1053 (2011).

7. Christianson, D. W. Structural biology and chemistry of the terpenoid cyclases. Chem. Rev. 106, 3412-3442 (2006).

8. Johnson, W. S., Semmelhack, M. F., Sultanbawa, M. U. S. \& Dolak, L. A. A new approach to steroid total synthesis. A nonenzymatic biogenetic-like olefinic cyclization involving the stereospecific formation of five asymmetric centers. J. Am. Chem. Soc. 90, 2994-2996 (1968).

9. Corey, E. J. \& Shouzhong, L. A short enantioselective total synthesis of Dammarenediol II. J. Am Chem. Soc. 118, 8765-8766 (1996).

10. Corey, E. J. Luo, G. \& Lin, L. S. A simple enantioselective synthesis of the biologically active tetracyclic marine sesterterpene Scalarenedial. J. Am. Chem. Soc. 119, 9927-9928 (1997). 11. Ishihara, K. Nakamura, S. \& Yamamoto, H. The first enantioselective biomimetic cyclization of polyprenoids. J. Am. Chem. Soc. 121, 4906-4907 (1999).

12. Bogenstatter, M., Limberg, A., Overman, L. E. \& Tomasi, A. L. Enantioselective total synthesis of the kinesin motor protein inhibitor Adociasulfate 1. J. Am. Chem. Soc. 121, 1220612207 (1999).

13. Pronin, S. V. \& Shenvi, R. A. Synthesis of highly strained terpenes by non-stop tail-to-head polycyclization. Nat. Chem. 4, 915-920 (2012).

14. Zhang, Q. \& Tiefenbacher, K. Terpene cyclization catalyzed inside a self-assembled cavity.

Nat. Chem. 7, 197-202 (2015).

15. Zhang, Q.; Rinkel, J.; Goldfuss, B.; Dickschat, J. S. \& Tiefenbacher, K. Sesquiterpene

cyclizations catalysed in-side the resorcinarene capsule and application in the short synthesis of isolongifolene and isolongifolenone. Nat. Cat. 1, 609-615 (2018).

16. Gutsche, C. D., Maycock, J. R. \& Chang, C. T. Acid-catalyzed cyclization of farnesol and nerolidol. Tetrahedron 24, 859-876 (1968).

17. Ohta, Y. \& Hirose, Y. Electrophile induced cyclization of farnesol. Chem. Lett. 1 263-266 (1972).

18. Polovinka, M. P. et al. Cyclization and rearrangements of farnesol and nerolidol stereoisomers in superacids. J. Org. Chem. 59, 1509-1517 (1994).

19. Susumu, K., Mikio, T. \& Teruaki, M. Biogenetic-like cyclization of farnesol and nerolidol to bisabolene by the use of 2-fuorobenzothiazolium salt. Chem. Lett. 6, 1169-1172 (1977).

20. Yadav, J. S., Thirupathaiah, B. \& Srihari, P. A concise stereoselective total synthesis of (+)artemisinin. Tetrahedron 66, 2005-2009 (2010).

21. Olah, G. A. \& Lukas, J. Stable carbonium ions. XXXIX. Formation of alkylcarbonium ions via hydride ion abstraction from alkanes in fluorosulfonic acid-antimony pentafluoride solution. Isolation of some crystalline alkylcarbonium ion salts. J. Am. Chem. Soc. 89, 2227-2228 (1967). 
22. Duttwyler, S.; Douvris, C.; Fackler, N. L. P.; Tham, F. S.; Reed, C. A.; Baldridge, K. K. \& Siegel, J. S. C-F activation of fluorobenzene by silylium carboranes: Evidence for incipient phenyl cation reactivity. Angew. Chem. Int. Ed. 210, 7681-7684 (2010).

23. Reed, C. A. $\mathrm{H}^{+}, \mathrm{CH}_{3}{ }^{+}, \mathrm{R}_{3} \mathrm{Si}^{+}$carborane reagents: When triflates fail. Acc. Chem. Res. 43, 121128 (2010).

24. Shao, B.+; Bagdasarian, A. L.+; Popov, S. \& Nelson, H. M. Arylation of hydrocarbons enabled by organosilicon reagents and weakly coordinating anions. Science 355, 1403-1407 (2017).

25. Hong, Y. J. \& Tantillo, D. J. Consequences of conformational preorganization in sesquiterpene biosynthesis. Theoretical studies on the formation of the bisabolene, curcumene, acoradiene, zizaene, cedrene, duprezianene, and sesquithuriferol sesquiterpenes. J. Am. Chem. Soc. 131, 7999-8015 (2009).

26. Hong, Y. J. \& Tantillo, D. J. Branching Out from the bisabolyl cation. Unifying mechanistic pathways to barbatene, bazzanene, chamigrene, chamipinene, cumacrene, cuprenene, dunniene, isobazzanene, iso- $\gamma$-bisabolene, isochamigrene, laurene, microbiotene, sesquithujene, sesquisabinene, thujopsene, trichodiene, and widdradiene sesquiterpenes. J. Am. Chem. Soc., 136, 2450-2463 (2014).

27. Reed, C. A. Carborane acids. New 'strong yet gentle' acids for organic and inorganic chemistry. Chem. Commun. 13, 1669-1677 (2005).

28. Kira, M. Hino, T. \& Sakurai, H. An NMR study of the formation of silyloxonium ions by using tetrakis[3,5-bis(trifluoromethyl)phenyl]borate as counteranion. J. Am. Chem. Soc. 114, 6697-6700 (1992).

29. Wigman, B.; Popov, S.; Bagdasarian, A. L.; Shao, B.; Benton, T. R.; Williams, C. G.; Fisher, S. P.; Lavallo, V.; Houk, K. N. \& Nelson, H. M. Strong bases and weak anions in catalytic C-H insertion reactions of vinyl carbocations. J. Am. Chem. Soc. 141, 9410-9144 (2019).

30. Kuprat, M., Lehmann, M., Shulz, A. \& Villinger, A. Synthesis of pentafluorophenyl silver by means of Lewis acid catalysis: Structure of silver solvent complexes. Organometallics 29, 14211427, (2010).

31. Olah, G.A., Molnár A. Hydrocarbon Chemistry, 2nd revised ed. Wiley Interscience, New York (2003)

32. Wu, Q. X., Shi, Y. P., J., Z. J. Eudesmane sesquiterpenoids from the asteraceae family. Nat. Prod. Rep. 23, 699-734 (2006).

33. Shippey, M. A. Dervan, P. B. Synthesis of cycloalkanes by intramolecular titanium-induced dicarbonyl coupling. J. Org. Chem. 42, 2655-2656 (1977).

34. Winkler, J. D., Sridar, V. \& Siegel, M. G. Ten-membered ring templates for stereoselective radical cyclizations. Tet. Lett. 30, 4943-4946 (1989).

35. Nevalainen, M. \& Koskinen, A. M. P. Synthesis of a 10-membered carbocycle by olefin metathesis. Angew. Chem. Int. Ed. 40, 4060-4062 (2001).

36. Hong, Y. J. \& Tantillo, D. J. The taxadiene-forming carbocation cascade. J. Am. Chem. Soc. 133, 18249-18256 (2011).

37. Dauben, W. G.; Hubbell, J. P.; Oberhansli, P. \& Thiessen, W. E. Acid-catalyzed cyclization of cembrene and isocembrol. J. Org. Chem. 44, 669-673 (1979).

38. Preckler-Angulo, C.; Genta-Jouve, G.; Mahajan, N.; de la Cruz, M.; de Pedro, N.; Reyes, F.; Iken, K.; Avila, C. \& Thomas, O. P. Gersemiols A-C and eunicellol A, diterpenoids from the arctic soft coral Gersemia fruticosa. J. Nat. Prod. 79, 1132-1136 (2016).

39. Faraldos, J. A., O’Maille, P. E., Dellas, N., Noel, J. P. \& Coates, R. M. Bisabolyl-derived sesquiterpenes from tobacco 5-epi-aristolochene synthase-catalyzed cyclization of (2Z,6E)farnesyl diphosphate. J. Am. Chem. Soc. 132, 4281-4289 (2010).

40. Yoder, R. A. \& Johnston, J. N. A case study in biomimetic total synthesis: polyolefin carbocyclizations to terpenes and steroids. Chem. Rev. 105, 4730-4756 (2005). 